\title{
Unusual Pneumonia Mimic
}

\author{
Joana Ricardo Pires ${ }^{1}$, Maria José Moreira ${ }^{1}$, Margarida Martins ${ }^{2}$, Clarinda Neves ${ }^{1}$ \\ ${ }^{1}$ Internal Medicine Department, Centro Hospitalar do Baixo Vouga, Aveiro, Portugal \\ ${ }^{2}$ General Surgery Department, Centro Hospitalar do Baixo Vouga, Aveiro, Portugal
}

\section{Received: 11/06/2019}

Accepted: 18/06/2019

Published: $17 / 07 / 2019$

How to cite this article: Pires JR, Moreira MJ, Martins M, Neves C. Unusual pneumonia mimic. EJCRIM 2019;6: doi:10.12890/2019_001181.

Conflicts of Interests: The Authors declare that there are no competing interests.

This article is licensed under a Commons Attribution Non-Commercial 4.0 License

\begin{abstract}
Disease in atypical organ locations can mimic other pathologies, hampering the right diagnosis. Such conditions may even be emergencies, like appendicitis. Subhepatic appendix is a very rare entity which may be caused by caecum dehiscence failure. The authors present the case of a 55-year-old immunocompetent man admitted to the Emergency Department with sepsis and severe hypoxaemia. Chest x-ray showed right lower lobe infiltrate, and community-acquired pneumonia was diagnosed. The patient was started on broad-spectrum antibiotics, but he continued to deteriorate and after 3 days developed abdominal complaints. Exploratory laparoscopy revealed an abscess caused by perforated subhepatic appendicitis.

Subhepatic appendicitis presents a diagnostic challenge and its clinical presentation may mimic that of other entities. This case highlights an atypical presentation, where the early development of inflammatory lung injury mimicked common pneumonia. Maintenance of a high index of suspicion and knowledge of these atypical locations is crucial.
\end{abstract}

\section{LEARNING POINTS}

- Control of the source of infection in sepsis is vital for survival.

- Acute inflammatory lung injury is common in sepsis and is correlated with clinical severity.

- A high index of suspicion and awareness of anatomical variants of the appendix are necessary for correct diagnosis of appendicitis mimicking other conditions.

\section{KEYWORDS}

Appendicitis, subhepatic appendix, sepsis, inflammatory lung injury

\section{CASE DESCRIPTION}

A 55-year-old man presented to the Emergency Department with pleuritic right chest pain for the previous 2 days, high fever $\left(40^{\circ} \mathrm{C}\right)$ and severe dyspnoea. He had no complaints of cough, sputum or abdominal pain. He had a history of hypertension and dyslipidaemia. At the time he was manager of the watering system of a public garden. He had no history of smoking or alcohol abuse, no previous surgeries, and no history of recent illness, travel or exposure to disease.

At admission, he had fever $\left(39.1^{\circ} \mathrm{C}\right)$, hypotension, tachycardia and an elevated respiratory rate. Crackles were heard over the right lower lung field, cardiac auscultation was normal, and there was right upper quadrant tenderness.

Arterial blood gases showed hypoxaemia with a $\mathrm{PaO}_{2} / \mathrm{FiO}_{2}$ ratio of 119 and hyperlactataemia (lactate $\left.2.5 \mathrm{mmol} / \mathrm{l}\right)$. Other laboratory results were: $\mathrm{Hb} 13.5 \mathrm{~g} / \mathrm{dl}$, normal white blood cell count and CRP $21.77 \mathrm{mg} / \mathrm{dl}$, normal renal function, LDH $157 \mathrm{U} / \mathrm{l}$ and normal plasma concentrations of hepatic enzymes. Chest x-ray demonstrated right lower pulmonary lobe infiltrate (Fig. 1) and abdominal ultrasonography revealed hepatic steatosis, a gallbladder without gallstones or signs of inflammation, and no peritoneal effusion. 
The echocardiogram was also normal. A diagnosis of sepsis from right lower lung pneumonia was made. Urine and blood cultures were collected, negative antigenuria and epidemiological circumstances ruled out Legionella infection, and piperacillin/tazobactam was prescribed.

The patient improved haemodynamically and was admitted to the Internal Medicine Department.

On the third day of antibiotic treatment, the patient still maintained fever and pleuritic pain, and developed worsening right upper quadrant pain with physical signs of peritonitis. Laboratory results showed increased CRP $(22.44 \mathrm{mg} / \mathrm{dl})$ and negative urine and blood cultures. Repeat abdominal ultrasonography showed densification of mesenteric fat in the right hypochondrium, revealing a poorly defined non-compressible $10 \mathrm{~mm}$ tubular structure associated with a heterogeneous hypoechogenic $23 \times 19 \mathrm{~mm}$ formation with irregular contours.

The patient underwent exploratory laparoscopic surgery which revealed subhepatic appendicitis with perforation and local abscess. Infection source control was implemented, but on the sixth day of antibiotic treatment, haematic and purulent fluid in the abdominal drain was observed. Multidrug-resistant Escherichia coli was isolated in the drainage fluid and antibiotic therapy was switched to meropenem. The patient improved rapidly and was discharged after 6 days of targeted antibiotic therapy.

\section{DISCUSSION}

An appendix in the subhepatic position is attributed to dehiscence failure of the caecum during embryonic development ${ }^{[1]}$. It is a rare anatomical variant whose incidence was estimated by Palanivelu et al. in a study with 7,210 patients to be $0.09 \%{ }^{[2]}$.

Cases of subhepatic appendicitis are rarely reported in the literature. Where described, patients usually have fever, nausea and abdominal pain in the right upper quadrant mimicking cholecystitis or liver abscess ${ }^{[2]}$. Much less common are patients with empyema, secondary to acute or postoperative appendicitis ${ }^{[3]}$. This phenomenon is explained by passage of bacteria from the abdomen to the thorax due to the pressure gradient, or the spread of infected fluids by the unilateral lymphatic flow across the diaphragm ${ }^{[3]}$.

However, in our case there was no lung infection but there was a local pulmonary inflammatory response secondary to sepsis mimicking pneumonia. This confounding effect delayed the diagnosis, leading to clinical worsening and perforation of the appendix and the subsequent abscess. Multiorgan failure is the most common cause of death in sepsis, and the lung is usually the first organ to be affected ${ }^{[4]}$. Recruitment of pre-activated granulocytes to the lung and granulocyte accumulation is associated with increased vascular permeability of the lung, and up-regulation of a variety of inflammatory factors in the lung ${ }^{[4]}$.

The authors emphasize the importance of being aware of anatomical variants of the appendix for the correct diagnosis of appendicitis and highlight the fact that acute inflammatory lung injury caused by sepsis is correlated with clinical severity and may mimic other conditions. 


\section{REFERENCES}

1. Rappaport WD, Warneke JA. Subhepatic appendicitis. Am Fam Physician 1989;39:146-148.

2. Palanivelu C, Rangarajan M, John SJ, Senthilkumar R, Madhankumar MV. Laparoscopic appendectomy for appendicitis in uncommon situations: the advantages of a tailored approach. Singapore Med J 2007;48:737-740.

3. Vasquez-Rios G, Calixto-Aguilar L, Pajuelo R, Alarcon W. Thoracic empyema as rare complication of an appendicular mass: a case study and review of the literature. Case Rep Pediatr 2018;2018:1-6.

4. Neumann B, ZantI N, Veihelmann A, Emmanuilidis K, Pfeffer K, Heidecke C-D, et al. Mechanisms of acute inflammatory lung injury induced by abdominal sepsis. Int Immunol 1999;11:217-227. 